\title{
Factores claves para la viabilidad de emprendimientos gastronómicos en momentos de crisis, en Guayaquil
}

\section{Key factors for the viability of gastronomic ventures in times of crisis, in Guayaquil}

MSc. Elba Calderón, Ing

Mgs. Pablo Ricardo San Andrés Reyes, Ing

Msc. Paola Jessica Plúa Terán, Econ.

Universidad Ecotec, Ecuador

Mgs. Nelly Sayenka Vergara Díaz.

Universidad de Guayaquil, Ecuador

Autor para correspondencia: nellyvergarad@gmail.com, ecalderon@ecotec.edu.ec, psanandres@ecotec.edu.ec,jplua@ecotec.edu.ec

Fecha de recepción: 10 de Septiembre de 2016 - Fecha de aceptación: 01 de Noviembre de 2016

Resumen: Los emprendimientos gastronómicos tienden a ser una de las opciones muy frecuentes al momento de desarrollar una idea de negocios en tiempos de crisis. Por ende, es importante desarrollar instrumentos que apoyen al emprendedor al logro de sus metas organizaciones, con el fin de poder incrementar la participación de mercado. Los emprendimientos pueden desarrollarse por la oportunidad de expandirse, tales como Kentucky Fried Chicken (1975), Pizza Hut (1982) y McDonalds (1997); que incluso fueron las primeras que entraron al Ecuador bajo esta modalidad. Pero también, se debe indicar varios negocios de emprendimiento gastronómico ecuatorianos que han logrado expandirse y desarrollar a través de cadenas o franquicias, tales como los Cebiches de la Rumiñahui y la Tablita del Tártaro. También pueden desarrollarse por necesidad, como ha sucedido en momentos de crisis a nivel mundial y en el Ecuador, así como en el año 1989 con el Café de Tere, cuya propietaria, la Señora Teresa de Castro decidió emprender una cafetería, como medio de incrementar sus ingresos, pues el sueldo que ganaba en aquella época no le abastecía. No todos los emprendimientos han logrado mantenerse en el mercado, expandirse e incursionar en Franquicias. Sus propietarios cometieron algunos errores comunes, tales como: poco uso de vestimenta de higiene, ubicación poco estratégica del local, control de calidad inadecuada, falta de herramientas tecnológicas, definir de manera incorrecta el concepto del restaurante, entre otros. Pero ¿Cómo lograr la sostenibilidad y desarrollo de estos emprendimientos gastronómicos?

Palabras claves: emprendimientos gastronómicos; crisis; mercado; participación de mercado; errores comunes

Abstract: Gastronomical ventures tend to be one of the most frequent options at the time of developing a business idea in crisis time. That's why it is important to create instruments to help the entrepreneur to reach his/her organizational goals and to increase the company's market share. Ventures can be developed by the opportunity to expand, such as: Kentucky Fried Chicken (1975), Pizza Hut (1982) y McDonalds (1997); even they are the first ones that arrived in Ecuador with this modality. But it is important to indicate that some gastronomical Ecuadorian ventures have expanded and developed through different locations or franchises, such as Cebiches de la 
Rumiñahui and Tablita del Tártaro. They can also be developed by necessity, as it has occurred in crisis times around the world and in Ecuador, like the year 1989 with Café de Tere which its owner, Mrs. Teresa de Castro decided to undertake a coffee shop to increase her incomes because her salary that used to earn was not enough for daily payments. Not all ventures have survived in the market, expanding or growing through franchises. Its owners made some common mistakes such as: No wearing hygiene clothes, wrong strategic location, inefficient quality control, lack of technology tools, defining in an incorrect way the concept of the restaurant, and others. But ¿How can be reached the sustainability and development of these gastronomical ventures?

Key words: gastronomical ventures; crisis; market; market share and common mistakes

\section{Introducción}

Ante la crisis económica que se vive en algunos países a nivel mundial, las personas se han visto en la necesidad de emprender nuevos negocios en su sociedad y una de las ideas más comunes son las relacionadas a emprendimientos gastronómicos.

El emprendedor es "El fundador de una nueva empresa, un innovador que rompe la forma tradicional de hacer las cosas, con rutinas establecidas. Debe ser una persona con dotes de liderazgo, y con un talento especial para identificar el mejor modo de actuar" (Schumpeter, 1934). Si bien es cierto, la innovación es fundamental para el desarrollo económico, las empresas deben de demostrar la factibilidad de una empresa en el mercado para asegurar una rentabilidad significativa y no invertir en activos cuya recuperación no vaya ser exitosa, especialmente en tiempos de crisis. Por tal motivo es muy importante conocer los pasos, metodología e instrumentos necesarios para demostrar la viabilidad de emprendimientos gastronómicos en tiempos en la cual el país este atravesando momentos complicados en la economía del país.

A través de esta investigación se determina los factores claves que necesitan los emprendimientos gastronómicos para viabilizar el negocio en momentos de crisis. A través del estudio de los componentes teóricos y científicos para el emprendimiento de negocios gastronómicos en Guayaquil y con el desarrollo instrumentos indispensables para invertir correctamente en emprendimientos gastronómicos en Guayaquil, se concluye con la determinación de factores necesarios para emprender.

Los emprendimientos gastronómicos necesitan implementar factores claves para asegurar la viabilidad en momentos de crisis.

¿Es suficiente que a través de la implementación de los factores claves se asegure la factibilidad del emprendimiento gastronómico?

\section{Explorar la importancia del problema}

El problema induce una nueva investigación, ya que existen negocios que fracasan por no tomar en cuenta ciertos factores indispensables para que el emprendimiento gastronómico tenga éxito. Por ende, al otorgar estas pautas y lineamientos se está capacitando al empresario que desea salir adelante, a pesar de que se hayan implementado políticas macroeconómicas ineficientes en el Ecuador. 


\section{Fundamentos teóricos}

En el año 2014, a pesar de que el índice de Actividad Emprendedora Temprana (TEA) en Ecuador se redujo de $36 \%$ a 32,6\% con respecto al 2013; es el más alto en América Latina. Los países que están en el segundo y tercer puesto son Perú y Chile con el 28,8\% y 26,8\% respectivamente. Los negocios de emprendimiento se enfocan en comidas, bebidas, tabaco y venta al por menor de productos especializados. Además existe un $22,8 \%$ de emprendedores por oportunidad, pero en cambio la Tea por necesidad tuvo un decrecimiento a 9,6\%, dato relacionado al año 2012 (GEM, 2014)

Este estudio demuestra que el nivel de emprendimiento se ha estabilizado a pesar los problemas económicos que ha atravesado el país durante los años 2013-2014. Las principales causas son: el temor al riesgo o por falta de educación. Por tal motivo, es indispensable que los futuros emprendedores conozcan, comprendan y sepan aplicar la metodología correcta para emprender un negocio, especialmente los relacionados a gastronomía, que son unas de las opciones que los ecuatorianos tienden a tomar en cuenta al momento de desarrollar una idea de negocio.

La técnica de Leideker consiste en identificar los factores críticos del éxito de los emprendimientos. Analiza los factores internos y externos que podrían afectar al negocio, además del impacto del mercado sobre las utilidades.

\section{Metodología}

El tipo de investigación a desarrollar es exploratorio, ya que se enfoca en temas poco estudiados y descubiertos, ya sea dentro de una estructura empresarial o en un mercado específico. En este caso se desea descubrir los principales factores de éxito que los negocios gastronómicos deben de tomar en cuenta al momento de emprender. A este estudio también se lo denomina cuantitativo, ya que los resultados arrojan porcentajes relacionados a las causas que llevaron al éxito a negocios gastronómicos ubicados en Guayaquil.

Además se desarrolló una investigación documental para recolectar datos relacionados a los indicadores económicos tales como: crecimiento del PIB alojamiento y comida, riesgo país, tasa de desocupados y aranceles. Luego de extraer dicha información, se aplicó un diseño no experimental de tipo transversal con muestreo no probabilístico (por conveniencia).

Esta metodología consiste en "analizar cuál es el nivel o estado de una o diversas variables en un momento dado o bien en cuál es la relación entre un conjunto de variables en un punto en el tiempo" (Dzul, M. s.f). También se lo define como "el diseño que recolecta datos en un solo momento, en un tiempo único. Su propósito es describir variables, y analizar su incidencia e interrelación en un momento dado. Es como tomar una fotografía de algo que sucede.” (Hernández, R.; Fernández C. \& Baptista P. 1991).

El plan de muestreo no probabilístico está conformado por los elementos del muestreo, la extensión y el tiempo. El primero se refiere al mercado meta en la cual se solicita la información, 
en este caso son los restaurantes que han tenido éxito en la ciudad de Guayaquil, quienes han tenido incursionando el negocio más de 10 años en el mercado. El segundo se define como el lugar donde se encuentran los datos del muestreo, con lo que se ha tomado en consideración la ciudad de Guayaquil en el Ecuador y el último se enfoca al tiempo en que se desarrolla la investigación, con lo que para efectos del mismo se lo aplicó durante la última semana del mes de mayo del año 2016.

A esto se sumó la técnica de Leidecker (1984) para identificar los factores críticos del éxito de los emprendimientos. Esta consiste en analizar el ambiente del negocio, la estructura de la industria, la opinión de expertos, la competencia, firma líder en la industria (bechmarking), evaluar la empresa, los factores temporales e intuitivos y el impacto de mercado sobre las utilidades.

Es importante recalcar que la comprobación de la aceptación de un producto o servicio a través de una investigación de mercados no es suficiente, ya que los costos y gastos que esa idea involucra podrían conllevar a una inviabilidad del proyecto. Además es necesario aplicar varios escenarios dependiendo de la situación económica en la cual se encuentre el país. Como en los actuales momentos estamos atravesando momentos de crisis en el Ecuador, es recomendable analizar un análisis de sensibilidad al cambio de la inflación como se muestra a continuación.

\section{Análisis de sensibilidad al cambio de la Inflación.}

\begin{tabular}{|c|c|c|c|c|c|c|c|c|c|}
\hline \multicolumn{3}{|c|}{ Análisis de sensibilidad al cambio de la inflación } & & & & & & & VAN \\
\hline AS11 & $6,59 \%$ & Flujo de caja & $(24,355,13)$ & $35,639,84$ & $19.150,64$ & $(78.693,03)$ & $27,604,34$ & $27.732,50$ & 0 \\
\hline Flujo Inicial & $(3,90 \%-3,35 \%)$ & Flujo de caja & $(24,355,13)$ & $35,639,84$ & $23.114,05$ & $(70.990,10)$ & $39.769,99$ & $45,422,14$ & $\$ 26.411$ \\
\hline & WACC & $12,00 \%$ & & & & & & & \\
\hline
\end{tabular}

Fuente: Banco Central recuperado Elaboración por: Autores

En el siguiente ejemplo se puede apreciar un flujo de caja proyectado a cinco años, en la cual la tasa de descuento es igual al 12\%, que equivale al Costo Promedio Ponderado de Capital (WACC), pero al analizar la sensibilidad al cambio de la inflación, se concluye que la inflación puede aumentar como máximo hasta un 6,59\% durante toda su proyecto para que el negocio siga siendo viable. Este análisis muchas veces es obviado, ya que se cree que si la tasa interna de retorno es mayor al costo de oportunidad, es aceptable la inversión, pero ciertas variables económicas pueden tener un cambio significativo en el tiempo, con lo que es aconsejable analizar nuevamente la viabilidad del negocio con el cambio variables tanto endógenas como exógenas.

El análisis de la industria analiza otros instrumentos, ya que es necesario investigar y estar conscientes de factores externos que podrían afectar al emprendimiento, ya sea a nivel nacional o internacional. Han existido algunos casos de negocio que emprenden sin tomar en cuenta las costumbres o estilos de vida que atraviesan ciertos territorios, con lo que al no medir 
estas variables el UONDI (Utilidad Operativa Neta después de Impuestos) se puede ver perjudicado en una medida tal que ni la cantidad de equilibrio se alcance vender.

Con la finalidad que el ingreso marginal de los productos y servicios que se ofrezcan el mercado tengan una tendencia creciente, es indispensable que aquellos cuenten con un valor agregado para que los consumidores los perciban como buena calidad. Por tal motivo, es necesario implementar un benchmarking y desarrollar un análisis minucioso de los competidores potenciales para desarrollar estrategias competitivas en el mercado.

\section{Resultados}

Los emprendedores seleccionados para la encuesta fueron: Walter Luzardo, propietario de Delicias Manabitas, Victor Herrara Chaparro, empleado antiguo del local La Palma, Teresa Castro, dueña del Café de Tere, Daniel Corozo Alarcón, del restaurante El Coroso. A través de la escala de Likert, utilizando una escala del 1 al 5 donde 5 es totalmente importante y 1 es nada importante, los emprendedores contestaron el nivel de importancia que han tenido los siguientes factores para el éxito de su negocio y los resultados fueron los siguientes:

\begin{tabular}{|c|c|c|c|c|c|}
\hline Criterios de evaluación & $\begin{array}{c}1 \text { (Nada } \\
\text { importante) }\end{array}$ & $\begin{array}{c}2 \text { (Poco } \\
\text { importante) }\end{array}$ & $\begin{array}{c}3 \\
\text { (Neutro) }\end{array}$ & $\begin{array}{l}4 \\
\text { (Importante) }\end{array}$ & $\begin{array}{l}5 \\
\text { (Totalmente } \\
\text { importante) }\end{array}$ \\
\hline Satisfacción laboral de los empleados & & & & 4 & 2 \\
\hline Localización del local & & & 1 & 1 & 4 \\
\hline El menú & & & & 1 & 5 \\
\hline Publicidad & 1 & & 1 & 2 & 2 \\
\hline Definición del concepto del restaurante. & 1 & & & 1 & 4 \\
\hline El prestigio de la organización. & & & 1 & & 5 \\
\hline El apoyo familiar & & 1 & & & 5 \\
\hline La recomendación de los clientes. & & & & & 6 \\
\hline Experiencia en el mercado & & 3 & & & 3 \\
\hline Opciones de financiamiento. & 2 & 2 & 1 & 1 & \\
\hline La perseverancia & & & & & 6 \\
\hline Empatía hacia el cliente & & & & 2 & 4 \\
\hline La apariencia del local & & & 1 & & 5 \\
\hline Calidad del producto & & & & & 6 \\
\hline Precio acorde al mercado & 1 & & & 2 & 3 \\
\hline Rapidez en la atención. & & 1 & 1 & 2 & 2 \\
\hline Apoyo de amigos & & 3 & & & 3 \\
\hline
\end{tabular}

Fuente: Elaboración propia

Factores más importantes de éxito para restaurantes gastronómicos 


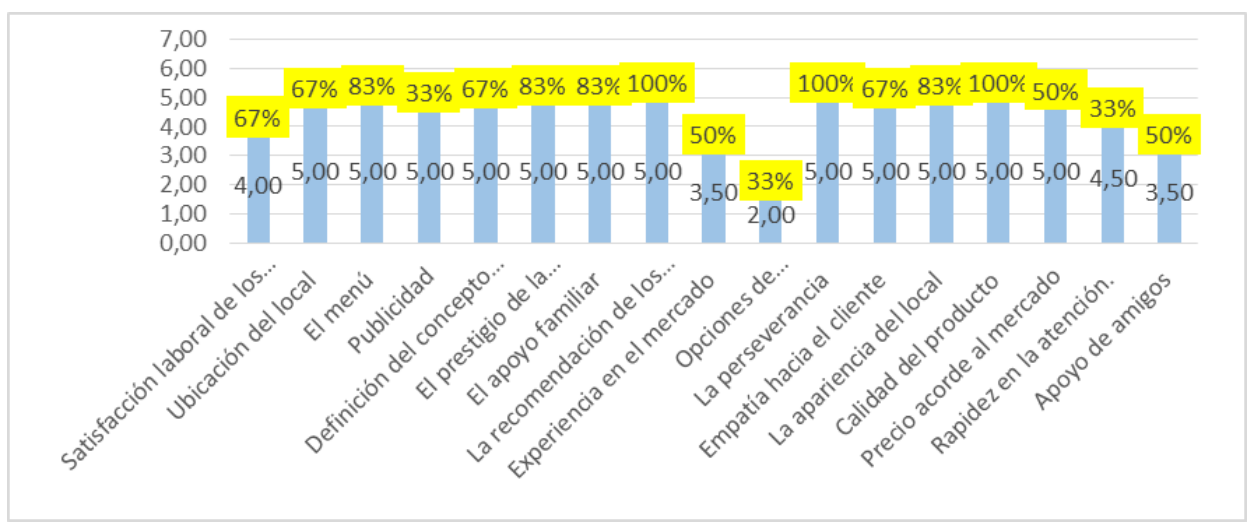

Fuente: Elaboración propia.

Tomando en consideración un rango entre $60 \%$ y $100 \%$ de los que contestaron importante y totalmente importante en cada uno de los factores de puede concluir que los factores más importantes que determinan el éxito de un negocio gastronómico son los siguientes:

$\checkmark$ Satisfacción laboral de los empleados,

$\checkmark$ Ubicación de local, el menú

$\checkmark$ Definición del concepto del restaurante,

$\checkmark$ Prestigio de la organización,

$\checkmark$ Apoyo familiar,

$\checkmark$ La recomendación de los clientes,

$\checkmark$ Perseverancia,

$\checkmark$ Empatía hacia el cliente,

$\checkmark$ apariencia del local y

$\checkmark$ Calidad del producto.

\section{Discusión}

El propósito del estudio fue corroborar posibles hipótesis sobre los factores críticos que contribuyen al éxito de los restaurantes de Guayaquil. Para los efectos se entrevistaron gerentes propietarios de establecimientos que alcanzaron éxito y permanencia de más de 10 años en el mercado. Dichos gerentes propietarios fueron entrevistados con un mismo conjunto de preguntas diseñadas de acuerdo a los factores críticos de éxito que se presentan en la industria restaurantera a nivel mundial y local para lo cual se tomó como base una investigación previa realizada en Texas y una aplicada en la ciudad de Quito, información que se expuso en el marco teórico. Las entrevistas fueron transcritas obteniendo de cada declaración información relevante para el estudio.

Aunque era común encontrar, a partir de la investigación secundaria, que algunos factores críticos eran representativos del mercado, sobre la base de las entrevistas se identificaron los siguientes tres factores críticos de éxito con el 100\% de importancia para los propietarios de restaurantes de la ciudad de Guayaquil. El primero es la calidad del producto, el segundo la recomendación de los clientes y el tercero la perseverancia. 
El primer factor crítico de éxito, la calidad del producto, se considera en función del sabor de los platos priorizando el uso de productos frescos y recetas propias para lograr una combinación firme y diferenciadora de la competencia.

El segundo factor, la recomendación de los clientes, es un factor crucial para la construcción de una base de clientes fiables para la utilización de marketing de boca a boca. Del mismo modo es necesario que los administradores tengan la capacidad de entender la psicología del cliente y puedan brindar un trato cálido y oportuno, capaz de seguir atrayendo más clientes.

El tercer factor es la perseverancia, estaría marcada por el hecho de que la mayoría de los encuestados tenían poca o ninguna experiencia en el negocio, motivo por el cual consideran que alcanzaron el éxito gracias a su determinación y constancia.

Se evidencia de la investigación que los propietarios de restaurantes identifican sus factores críticos de éxito, sin embargo no demuestran estar utilizando los beneficios que estos aportan a su negocio como una herramienta estratégica, a pesar que la dinámica del mercado señala que algunos factores claves como la apariencia del local, ubicación y menú pueden ser comparados con el competidor con el fin de proporcionar una ventaja competitiva a largo plazo. Tampoco están creando vínculos entre estos factores y su contribución a la gestión del rendimiento, quizás sea debido a la complejidad de la medición de algunos de los factores; sin embargo, es también probable que sea un reflejo del enfoque simplista a la medición del rendimiento que se ha establecido por esta investigación

A pesar de ello, los nuevos emprendedores de negocios gastronómicos o sus administradores pueden utilizar los resultados de la investigación como una referencia para comprender los puntos críticos a potenciar durante el proceso y fijar metas y estrategias sostenibles.

\section{Conclusiones}

En base al objetivo general de la presente investigación fue posible llegar a las siguientes conclusiones:

Se realizó la entrevista a expertos bajo el diseño no experimental del tipo transversal. A través de la busca de emprendedores se pudo establecer que en Guayaquil existen empresarios con más de 10 años de experiencia en el mercado gastronómico.

A través de las técnicas aplicadas se pudo determinar que los factores de éxito de las empresas guayaquileñas está en el Servicio al cliente, menú, apoyo familiar, el prestigio de la organización, la recomendación de los clientes, la perseverancia, la apariencia del local y la calidad del producto.

Los empresarios demostraron tener la buena predisposición de transmitir su experiencia a los jóvenes emprendedores. 
Los emprendedores pueden analizar la viabilidad de un negocio través de la aplicación de la técnica de Leidecker. Es necesario desarrollar un análisis de sensibilidad de la inflación en los flujos proyectados en tiempos de crisis.

\section{Agradecimientos}

Agrademos a los profesores de la Facultad de Ciencias Económicas y Empresariales de la Universidad Ecotec de Guayaquil, por sus sabias asesorías de investigación para culminación exitosa de la ponencia "Factores claves para la viabilidad de emprendimientos gastronómicos en momentos de crisis, en Guayaquil“. Además a Walter Luzardo, propietario de Delicias Manabitas, Victor Herrara Chaparro, empleado antiguo del local La Palma, Teresa Castro, dueña del Café de Tere y Daniel Corozo Alarcón, del restaurante El Coroso por su valiosa información sobre su experiencia en el mercado

\section{Bibliografía}

Angelelli, P. \& Moori, V. (s.f.). Desarrollo Emprendedor América Latina y Experiencia Internacional. Recuperado http://www.emprendedorxxi.coop/Pdf/Desarrollo_Emprendedor\%5B1\%5D.pdf

Ashar, M., Blach, G., Mandabach, K. \& Van Leeuven, D. (2011). Restaurant Viability: Operations Rating of Contributing Success Factors. Journal of culinary science. Taylor \& Francis Group. Obtenido el 15 de enero del 2013.

Banco Central del Ecuador (2016).

Borja, J. \& Rivadeneira, L. (2013). Factores para el logro del éxito de la industria de restaurantes: Caso relacionado con restaurants independientes de la provincial Pichincha, Ecuador. (tesis de pregrado). Universidad San Francisco de Quito, Pichincha, Ecuador. Recuperado de file:///C:/Users/Paopa\%20plua/Downloads/Factores\%20CR\%C3\%8dticos\%20\%C3\%89xito $\%$ 20restaurantes\%20quito.pdf

Hoang, P. (2007). Business and Management. (1era. ed.). Victoria, Australia: IBID Press

Lasio, V., Caicedo, G., Ordeñana, X. \& Izquierdo, Edgar (2014). Global Entrepreneurship Monitor. Recuperado de http://www.espae.espol.edu.ec/images/documentos/publicaciones/libros/gemecuador2014.pdf

Mullins, Walker Boyd. (2005). Marketing estratégico (págs. 224-246). México: Mc Graw Hill.

Navarro, P. (2005). Finanzas corporativas. Barcelona: Bresca Editorial

Velasco, Gabriela (2011). Factores críticos del éxito empresarial: Una aplicación en las empresas restauranteras en el Municipio de Juárez del Estado de Chihuahua. (Congreso Internacional de Contaduría, Administración \& Informática). Universidad Autónoma de ciudad Juárez México, Juárez, México. 